\title{
Criminologie
}

\section{Les normes de l'intervention auprès des jeunes mésadaptés : Bilan des écrits}

\section{Maurice Cusson, Danielle Laberge-Altmejd et Hélène Grenier}

Volume 11, numéro 2, 1978

Normes et politique criminelle

URI : https://id.erudit.org/iderudit/017091ar

DOI : https://doi.org/10.7202/017091ar

Aller au sommaire du numéro

Éditeur(s)

Les Presses de l'Université de Montréal

ISSN

0316-0041 (imprimé)

1492-1367 (numérique)

Découvrir la revue

Citer cet article

Cusson, M., Laberge-Altmejd, D. \& Grenier, H. (1978). Les normes de

l'intervention auprès des jeunes mésadaptés : Bilan des écrits. Criminologie,

11(2), 42-75. https://doi.org/10.7202/017091ar d'utilisation que vous pouvez consulter en ligne. 
LES NORMES DE L'INTERVENTION AUPRES

DES JEUNES MESADAPTES

BILAN DES ECRITS '

Maurice Cusson

Danielle Laberge-Altmejd en coll. Hélène Grenier

Les normes de l'intervention auprès des mineurs inadaptés (délinquants et enfants en danger) ce sont des règles qui définissent ce qui devrait être fait dans les agences correctionnelles pour jeunes.

Ce document est constitué d'une discussion sur l'usage de telles normes et d'une sélection de celles qui ont été jugées les plus intéressantes lors d'une analyse des écrits spécialisés. Ce choix des normes est la partie la plus importante de cet article. Le lecteur doit garder à l'esprit qu'il ne s'agit pas d'un travail définitif, mais d'un point de départ à une discussion informée. Plus important encore, même si elles peuvent en avoir l'air, ces normes ne doivent pas être considérées comme des dogmes mais bien comme des éléments de solution à des problèmes qui se posent, ici comme ailleurs, comme des options possibles.

Les grandes divisions de cet article comprennent une première partie, où on traite de la nature et de l'utilité des normes ou standards, puis de la nature des normes, de leur signification pour l'intervention de leur utilité et des risques qu'elles comportent, ainsi que de la démarche et de la méthode de travail.

Dans la deuxième partie, on trouvera le bilan des écrits, soit le recueil des normes, les principes et les objectifs, la nature des décisions et des services ainsi que du cadre de l'intervention et de l'organisation.

\section{La nature des normes}

Les standards ou normes ${ }^{2}$ de l'intervention auprès des jeunes mésadaptés sont des règles qui indiquent les lignes d'action

1. Ce travail a été rendu possible grâce à une subvention de la Société canadienne de criminologie.

2. Les termes \& norme $»$ et * standard \$ sont ici considérés comme synonymes et employés exactement dans le même sens. 
que devraient adopter les institutions, les foyers de groupe, les services de probation et autres agences ayant pour clients des jeunes qui ont des problèmes d'adaptation tels que la délinquance. Ces normes établissent les conditions à respecter pour offrir aux clients et à la société les meilleurs services possibles. Elles se présentent comme des énoncés brefs sur ce qui devrait être et, à ce titre, fournissent un modèle auquel on compare une pratique ou une action. A la condition d'être suffisamment précise, la norme ou standard offre un critère, un point de référence à celui qui veut évaluer les politiques et les interventions des praticiens.

Le standard définit l'excellence telle qu'on l'entend actuellement. Il décrit la meilleure manière d'agir avec les jeunes délinquants. Pour ce faire, les standards sont élaborés en tenant compte des connaissances scientifiques (en criminologie, en psychologie, en éducation, en psychiatrie, etc.), des principes de politique criminelle, des exigences de justice, d'humanité, de l'expérience des praticiens. En somme, les standards réalisent une synthèse des connaissances et de l'expérience dans le but de définir et de guider l'action. À ce titre, ils ne sont pas immuables et doivent subir les modifications qu'entraînent les progrès de la science et l'évolution de la pratique. Les normes sont formulées par des spécialistes dont la compétence est reconnue, auxquels on demande quelles sont les politiques, procédures, méthodes, pratiques qui serviraient le mieux les intérêts des jeunes et ceux de la société.

Les standards peuvent varier selon le degré d'importance qu'on leur accorde. Il peut exister des normes \& minimales \$ qui définissent les exigences jugées essentielles, qui sont alors obligatoires, et d'autres normes, « optimales », qui indiquent la meilleure solution possible dans l'état actuel des connaissances, ces dernières sont alors de l'ordre du désirable.

Dans l'industrie, où les standards existent depuis bien plus longtemps que dans les services correctionnels, on les regroupe en quatre catégories (cf. Encyclopedia Britannica) :

1) Techniques, s'ils réfèrent aux produits, au matériel, à l'équipement ;

2) Administratifs (managerial), s'ils concernent les pratiques et les procédures relatives à l'administration ; 
3) Substantiels (substantive), s'ils s'attachent à ce qui devrait être fait ;

4) Procéduraux, s'ils décrivent comment une tâche devrait être accomplie.

Étant principalement intéressés à l'intervention auprès des jeunes délinquants, nous nous sommes attachés presque exclusivement aux deux dernières catégories, soit les standards «substantiels > et "procéduraux ». Nous ne nous sommes intéressés aux normes administratives qu'en autant qu'elles concernent l'intervention criminologique. Finalement, les standards techniques, dont l'importance n'est pourtant pas niée, n'entrent pas dans le cadre de cette étude.

Par «l'intervention auprès des jeunes mésadaptés », nous entendons l'ensemble des mesures destinées aux jeunes délinquants et aux enfants en danger qui passent devant les tribunaux. Ceci inclut les centres de détention, les institutions de réadaptation, les foyers de groupe, les services de probation, de réadaptation communautaire, de réinsertion sociale, etc. Sont exclus de notre propos, les services de prévention ainsi que les tribunaux pour mineurs.

\section{Signification des normes pour lintervention}

Les standards correctionnels existent depuis longtemps (en 1870, l'American Correctional Association publiait une déclaration de principes qui peut être considérée comme le premier recueil de standards en Amérique du Nord), mais ce n'est que depuis quelques années que l'on a entrepris un effort d'envergure pour élaborer des standards susceptibles d'être appliqués dans les services correctionnels. Aux Etats-Unis, la National Advisory Commission on Criminal Justice Standards and Goals commençait, en 1973, à publier une série de rapports alors qu'était créée en 1974 une Commission on Accreditation for Correction. Dans le domaine hospitalier, la Joint Commission on Accreditation of Hospitals publiait en 1971 et 1973 des recueils de standards remarquables qui peuvent nous inspirer, même s'ils sont destinés à des clientèles différentes. Au Québec, l'Association des centres d'accueil travaille sur des standards susceptibles de s'appliquer dans les établissements qui en sont membres. 
Dans d'autres provinces, des efforts semblables sont entrepris dont notamment l'Ontario et l'Alberta.

Ces différents projets émanant d'organismes très divers, convergent dans le même sens. C'est un véritable mouvement qui a une signification majeure parce qu'il répond à des besoins pressants tout particulièrement dans le domaine de l'action criminologique. En effet, depuis quelques temps, la criminologie est dans une impasse. L'idéal thérapeutique et la criminologie clinique n'ont engendré que déceptions et désillusions. Par le traitement, on a cru pouvoir convertir les délinquants en utilisant des méthodes mystérieuses, quasi magiques. Or, les évaluations rigoureuses nous apprennent maintenant qu'on ne réussit pas à transformer de façon durable les personnes, surtout quand on agit contre leur gré. La criminologie clinique s'est alors confinée de plus en plus dans le perfectionnement des méthodes de diagnostic et d'évaluation, démarche stérile s'il n'existe pas de traitement efficace à prescrire. L'échec des cliniciens a engendré une réaction nihiliste : la criminologie radicale. Jeunes et moins jeunes turcs, redécouvrant la dimension répressive de la réaction sociale, emploient un jargon démagogique pour nous inviter à nous croiser les bras en nous prêchant la «non-intervention radicale », quand ce n'est pas la révolution permanente. Entre une criminologie clinique qui tourne à vide et une criminologie radicale qui érige la démission en idéal, le mouvement discret des normes et standards prépare l'avenir en définissant les critères de l'intervention et en forgeant par le fait même les outils de ceux qui travaillent auprès des jeunes délinquants.

\section{Utilité des normes, les risques qu'elles comportent}

En effet, les normes telles qu'elles se développent présentement peuvent donner à l'homme d'action à la fois une méthode et une orientation.

Tout d'abord, elles clarifient les règles du jeu. Trop souvent les praticiens sont plongés dans l'empirisme et dans les solutions à court terme. Leurs obligations sont fioues, imprécises. Sur des problèmes fondamentaux, on les laisse dans l'indétermination alors que dans d'autres secteurs on les enserre dans des règlements tatillons. Les normes et standards viennent à propos pour définir avec précision et cohérence les programmes et les interventions. Ils fournissent un guide d'action détaillé. Les res- 
ponsabilités des intervenants deviennent claires et, en conséquence, les risques d'abus, d'arbitraire et d'incohérence en sont réduits d'autant.

Deuxièmement, les standards rendent possible une opérationnalisation systématique des principes de politique criminelle, favorisant par le fait même leur implantation. On évite ainsi cette coupure entre la théorie et l'action qui a été une véritable plaie dans notre domaine. Les principes et objectifs généraux sont alors traduits en programmes précis, en décisions concrètes, en règles de fonctionnement. Il est à propos ici de faire remarquer que les standards contemporains ne nous apportent pas seulement un contenant, ils véhiculent en même temps un contenu, aussi bien sur le plan des objectifs que sur celui des moyens. Au niveau des objectifs, les meilleurs recueils de standards abandonnent cette fixation sur un objectif unique et englobant; ils nous proposent en contrepartie une diversité d'objectifs et de principes complémentaires : justice, humanité, intégration sociale, intervention minimale, éducation, modification du comportement, etc. Sur le plan des moyens, les recueils de standards nous invitent à contrôler la qualité du travail à tous les niveaux : dans les services offerts, dans le processus de prise de décision, dans le milieu de vie du client, dans ses relations avec la société en général, dans les règlements, les sanctions, les mesures de contrôle, etc.

Troisièmement, les standards fournissent les critères nécessaires à l'accréditation et à l'évaluation. Traditionnellement, l'évaluation criminologique s'est contentée de mesurer un résultat unique et lointain : la récidive tout en ignorant la diversité des objectifs poursuivis, de même que la qualité des moyens utilisés. Avec les standards, une évaluation plus complète, plus nuancée, plus susceptible de fournir une rétroaction rapide au praticien devient possible. Des évaluations d'un nouveau genre émergent et facilitent l'auto-évaluation, l'évaluation continue et l'accréditation.

Finalement, les standards encouragent et stimulent ceux qui travaillent auprès des délinquants à élever la qualité des services, d'abord parce qu'ils définissent l'excellence mais surtout parce que cette définition du désirable est confrontée systématiquement à la réalité dans le processus d'évaluation et d'accréditation. Les principes cessent alors d'être des vœux pieux, et permettent au praticien de se juger, de se corriger et de progresser. 
Malgré l'intérêt des normes, il ne faut pas sous-estimer les risques qu'elles comporteraient si elles étaient mal conçues et mal utilisées.

Il faut d'abord reconnaître l'énorme difficulté que les responsables d'établissements pour jeunes délinquants éprouvent à offrir des services tout simplement décents. $\mathrm{Si}$, dans ces conditions, on exige d'eux qu'ils se conforment à des standards trop élevés, leurs difficultés, déjà grandes, risqueraient de devenir insurmontables : problèmes de contrôle sur les jeunes, roulement du personnel, recrutement insuffisant, conflits de relation de travail, démissions chez les cadres, etc. $\mathrm{Si}$, par des standards irréalistes, on met une pression trop forte sur les directeurs et les praticiens, on verra apparaître inévitablement des pratiques comme le a dumping ». Car un des moyens les plus faciles pour avoir l'air d'améliorer le fonctionnement d'un service correctionnel, est de manceuvrer pour avoir des clients meilleurs, plus faciles, plus prêts à coopérer, plus soumis. Le «dumping * est un moyen d'avoir des sujets faciles : il s'agit de refuser ou d'expulser ceux qui sont un peu trop compliqués. Et alors, le niveau de fonctionnement ne peut que monter, non pas parce que les services sont meilleurs, mais parce que les clients coopèrent mieux. Cette pratique qui conduit à une pseudo-amélioration peut être la conséquence de l'utilisation des standards excessifs et mal compris. C'est pourquoi il est essentiel que ceux-ci soient réalistes. Ils doivent tenir compte du niveau actuel des agences, des capacités des praticiens, du degré de difficulté de la clientèle, des ressources matérielles disponibles, etc.

Il est évident que les standards ne doivent pas \& coller * purement et simplement aux pratiques existantes, car ils n'auraient alors aucune utilité. Ils doivent décrire un idéal mais un idéal accessible. Pour conserver ce réalisme nécessaire, la participation de ceux qui sont dans l'action est indispensable. Directeurs, cadres et exécutants doivent être impliqués dans la rédaction et l'acceptation des standards qu'on utilisera pour les évaluer. Un dialogue doit s'établir entre théoriciens et praticiens pour que les standards conservent un équilibre entre l'idéal et la réalité, entre le désirable et le possible.

S'il peut être désavantageux de définir des standards irréalisables, l'imposition coercitive de standards pourrait être catastrophique. Aux Etats-Unis certains juges et certaines autorités ont 
prétendu obliger des institutions à se conformer à des standards de traitement et, en définitive, ils ont fait plus de tort que de bien (Miller, 1977) : les praticiens, de concert avec leurs supérieurs, refusaient de donner les informations nécessaires à l'application des standards, ils falsifiaient les rapports et faisaient de l'obstruction. La rotation du personnel et des administrateurs augmentait et les clients les plus difficiles étaient refusés ou expulsés. Dans l'état actuel de nos connaissances et de nos ressources, il est possible de définir des standards " minima » dans un petit nombre de secteurs où ils s'imposent (locaux, alimentation, punitions, discipline, etc.), mais, pour le reste, les standards ne devraient pas être imposés et rendus obligatoires. Le processus d'accréditation devrait se faire, comme dans les hôpitaux, sur une base volontaire. Plutôt que de miser sur la crainte de sanctions négatives, il nous semble préférable de tabler sur la stimulation et l'émulation que déclenchera un système d'accréditation volontaire et prestigieux.

Enfin, les standards risquent d'accentuer la bureaucratisation des agences. S'ils sont trop détaillés, ils restreindront inutilement la marge de manouvre de ceux qui prennent les décisions, ils ouvriront la porte à la paperasserie et aux contrôles tatillons. $\mathrm{Si}$, en outre, ils sont considérés comme des règles immuables, édictées une fois pour toutes, ils empêcheront l'innovation et les changements. C'est pourquoi il faut pratiquer la parcimonie et préférer un petit nombre de standards, sur des points essentiels ou stratégiques, à un grand nombre portant sur des détails. Il faudra aussi déterminer la durée d'application des standards, puis les réviser et les modifier périodiquement en tenant compte de l'évolution des connaissances et des leçons de l'expérience.

\section{Démarche et méthode de travail}

L'objectif poursuivi était de parcourir les écrits sur les normes susceptibles de s'appliquer aux agences correctionnelles pour jeunes afin d'en recueillir celles qui étaient valables et pertinentes. Vingt-six publications spécialisées, surtout des recueils de normes, ont été retenues. Toutes les normes contenues dans ces ouvrages qui, à première vue semblaient intéressantes ont été recueillies, il y en avait 749. Par la suite, un long travail d'élimination, de regroupement et de classification nous a permis d'éliminer 310 standards. C'est à partir des 439 normes qui restaient 
que nous en sommes arrivés aux 239 normes que comprend le recueil qui suit.

Un effort particulier a été fait pour classer les normes dans le but de leur donner l'organisation la plus rigoureuse et la plus logique possible. Quatre systèmes de classification ont été utilisés successivement pour en arriver à l'organisation finale qui est faite de 18 sections regroupées en cinq grandes têtes de chapitre : 1) Les principes et objectifs, 2) Les décisions, 3) Les services, 4) Les cadres de l'intervention, 5) L'organisation. Cette mise en ordre devrait contribuer, nous semble-t-il, à nous faire sortir de la confusion qui règne actuellement lorsqu'il s'agit de définir l'intervention auprès des jeunes délinquants.

Le processus de sélection des normes s'est fait de façon intuitive. Il a été néanmoins guidé par les considérations suivantes :

- Les normes retenues ne devaient naturellement entrer en contradiction avec ce que les recherches, l'expérience et les réflexions nous ont appris sur les conséquences de l'intervention. C'est pourquoi certains standards ont été éliminés parce qu'ils n'étaient pas valables, compte tenu des connaissances actuelles.

- Les normes les plus séduisantes sont en équilibre entre l'utopie et la réalité. Une norme complètement utopique n'est pas intéressante parce que non réalisable. Par ailleurs, une norme qui ne fait que décrire la réalité ne l'est pas tellement plus : elle ne propose pas d'amélioration, elle ne fait que rendre compte d'un état de fait. Si on veut que les standards incitent, stimulent les praticiens à améliorer leurs services, il faut développer des standards qui soient toujours quelques pas plus loin que ce qui se fait à un moment donné. Malgré tout, les acquis doivent être maintenus, c'est pourquoi, doivent aussi être conservées les normes qui rappelleront à celui qui est dans l'action la nécessité de maintenir les pratiques qui ont fait leur preuve et dont la valeur est reconnue.

- Les standards doivent porter sur des questions, soit essentielles, soit importantes. Ceux qui portent sur des détails, des questions mineures, ne méritent pas d'être retenus. Ils ne servent qu'à limiter l'initiative des gens et à bureaucratiser encore plus les agences.

Certaines normes sont applicables dans un contexte comme celui du Québec, d'autres ne le sont pas. Nous n'avons retenu que les standards qui semblaient s'adapter à la situation des 
établissements du Québec, à leur fonctionnement, à leurs valeurs, etc.

Finalement, les normes doivent être formulées avec précision et clarté. Les propositions vagues, les généralités, les tartes à la crème, les souhaits pieux étaient rejetés dans notre processus de choix des standards les plus adéquats.

Le recueil de normes qui suit est loin d'être un ouvrage achevé. Il n'est qu'une revue systématique des écrits. Or, les recueils de normes actuels sont loin d'être complets. Par conséquent, le présent recueil ne fait que refléter cet état de choses, il est incomplet. Malgré tout, dans certains secteurs, l'éventail des problèmes est assez bien couvert. Notons en particulier : les décisions à l'admission, les règlements, sanctions et mesures de contrôle (sauf pour l'isolement), le personnel et les dossiers. D'autres secteurs sont pauvres : les relations personnel-client, le milieu de vie, les loisirs, le traitement. Ces secteurs où la littérature est insuffisante pourraient être développés et enrichis en s'inspirant des procédures et modèles d'intervention qui existent dans les centres d'accueil du Québec et qui pourraient être formulés en termes de normes. Ce travail n'a pas été fait et il devrait l'être. En particulier, l'expérience et les pratiques des psychoéducateurs pourraient être ainsi codifiées avec profit.

Les normes qui suivent sont insuffisamment commentées et explicitées. Seuls quelques commentaires jugés indispensables ont été rapidement jetés sur le papier mais c'est insuffisant et cela donne à notre recueil une sécheresse et une allure rigide qu'il ne devrait pas avoir.

Compte tenu de ce qui précède, il est possible d'indiquer rapidement les étapes du travail qui resterait à faire pour en arriver à un produit fini :

1) Combler les lacunes, surtout en s'inspirant de l'expérience et des pratiques développées au Québec, en particulier dans les centres d'accueil.

2) Commenter et expliciter les normes de façon à en donner la signification et la justification.

3) Consulter des experts, aussi bien praticiens que théoriciens pour modifier, éliminer les standards existants et en développer de nouveaux. 
4) Adapter les standards aux différents types d'établissements auxquels ils devront s'appliquer (centre de détention, institution de réadaptation, centre de transition, foyer de groupe, service de probation, etc).

5) Faire participer les praticiens à qui pourraient s'appliquer ces normes et obtenir leur adhésion.

6) Pré-expérimenter les standards dans certains établissements afin de savoir s'ils sont utilisables.

7) Reformuler, modifier, réviser de façon continue les normes dans un processus de remise en question permanente qui s'inscrit au cœur même de la démarche d'accréditation.

Dans le recueil de normes, les sources sont indiquées de façon un peu spéciale. Après chaque norme, on trouve le pays, l'état ou la province, où a été publié le recueil cité. Les références complètes sont données à la fin du texte. Les normes qui reproduisent fidèlement nos sources sont suivies de la référence alors que les références des normes qui existent dans la littérature sont suivies du signe : cf.

\section{Bilan des écrits : recueil des normes}

Les normes qui suivent pourraient s'appliquer à divers types d'établissements pour jeunes mésadaptés sociaux : centre de détention (sécuritaire), centre de transition (hébergement), centre de réadaptation, service de probation, service de réadaptation communautaire, foyer de groupe, centre de jour, etc. Sauf dans quelques cas, le type d'établissement auquel pourrait s'appliquer une norme n'est pas indiqué. Les variations d'un établissement à l'autre sont trop grandes pour qu'il soit possible de le faire. Par ailleurs, les agences ont des vocations particulières et décident d'offrir tel service et non pas tel autre. Ce qui veut dire qu'une série de normes qui s'appliquent à un service particulier (par exemple : l'emploi et le marché du travail) ne s'appliqueraient pas à un établissement qui n'offrirait pas ce service. Il peut arriver aussi qu'une norme ne puisse s'appliquer à tel établissement qu'avec des modifications. Finalement, certains standards peuvent être contre-indiqués dans certains cas.

Une mise en garde essentielle : ces normes ne sont pas des dogmes. Ce sont des guides de l'action, une espèce de répertoire 
de solutions aux problèmes qui se posent dans les établissements pour jeunes mésadaptés. Il est utile d'être informé des choix qui ont été faits ailleurs, ceci ne veut pas dire qu'il faut les copier. Plusieurs normes paraîtront au lecteur discutables : excellent ! C'est qu'elles méritent d'être discutées. Espérons que les débats seront profitables. À ceux qui, au Québec, travaillent auprès des jeunes mésadaptés, ce recueil apportera souvent des points de vue différents de ceux auxquels ils sont habitués. S'ils sont complémentaires, c'est tant mieux, s'ils sont contradictoires, ce sera une occasion de s'arrêter, de réfléchir et, en toute liberté, d'adapter la solution qui leur paraîtra la meilleure.

\section{PRINCIPES ET OBJECTIFS}

\section{1 Énoncé de politique}

1.1.1 L'établissement dispose d'une description écrite des services offerts et de ses politiques (cf. États-Unis 4, 8, 9, Ontario 1, Wisconsin 1).

1.1.2 Cette description inclut les points suivants :

- objectifs

- principes de base

- conditions d'admission et clientèle

- durée de séjour et/ou conditions de départ

- procédures pour adapter le programme aux besoins individuels

- services offerts

(cf. États-Unis 4, 8, 9, Ontario 1, Wisconsin 1).

1.1.3 Le document décrivant les services offerts et la politique de l'établissement est accessible aux clients, au personnel, aux services de référence ainsi qu'au public en général (cf. États-Unis 4, 8, 9, Ontario 1, Wisconsin 1).

1.1.4 Le programme et les services offerts par l'agence correspondent étroitement à ses objectifs, principes et énoncés de politique (États-Unis 4).

1.1.5 Les services offerts répondent à des besoins clairement identifiés (États-Unis 4).

1.1.6 L'établissement dispose d'un manuel des politiques et procédures qui décrit les interventions, mesures, moyens et processus utilisés pour réaliser ses objectifs (États-Unis 9).

1.1.7 Les procédures de l'établissement correspondent aux : principes et objectifs de l'établissement

besoins des clients

théories et principes acceptés

ressources disponibles

(États-Unis 9).

1.1.8 L'établissement fait en sorte que le jeune ait une connaissance claire de la nature de la transaction qui le lie avec l'établissement (Ontario 2).

1.1.9 Cette entente contractuelle précise nettement, entre autres choses :

- la durée du service ou du séjour

- les objectifs de l'intervention 
- les droits du client

- les obligations et les sanctions s'y rattachant

- les services offerts

- les activités

- la personne responsable du jeune (tuteur) (cf. Ontario 2).

\subsection{Objectifs}

\section{Introduction}

Les objectifs poursuivis par un établissement ou un service peuvent être fort divers. Il arrive d'ailleurs fréquemment que pour un jeune donné l'intervention ait plus d'un but. Voici une liste de ces buts :

1) Compléter la formation scolaire du jeune

2) Donner une formation professionnelle au jeune

3) Favoriser le développement optimal du client et, en particulier : a) le développement affectif ; b) le développement social ; c) le développement à la communication

4) Valoriser, développer l'estime de soi du jeune

5) Aider à l'intégration sociale du client dans les secteurs de la famille, l'école, le marché du travail et les loisirs

6) Corriger et modifier les comportements problématiques

7) Répondre aux besoins spéciaux qui se manifestent chez les jeunes ayant des problèmes d'adaptation

8) Héberger convenablement le jeune

9) Protéger la société contre les actes anti-sociaux de jeunes délinquants

10) Empêcher l'agir dêlinquant ou apprendre au jeune à le contrôler (cf. Québec 1, États-Unis 8, États-Unin 3).

\section{Définitions}

Développement social : Développement de la capacité d'entrer en relation avec autrui et de maintenir des relations satisfaisantes avec son milieu social. Capacité de jouer les rôles appropriés. Développement de l'autonomie dans ses rapports avec les gens et la société (cf. Etats-Unis 9).

Développement affectif : Développement des sentiments et des émotions. Inclut le développement des comportements qui sont reliés aux intérêts, aux émotions, aux valeurs (États-Unis 9).

Développement à la communication : Développement de la capacité à transmettre des significations à autrui de façon verbale ou non-verbale, permettant ainsi de maintenir le contact et de répondre au milieu social (États-Unis 9).

1.2.1 L'établissement a une description écrite de ses objectifs (États-Unis 9).

1.2.2 Les objectifs correspondent à des besoins identifiés chez la clientèle de l'établissement (États-Unis 9).

1.2.3 Les objectifs sont reliés aux objectifs généraux du réseau dont fait partie l'établissement (États-Unis 9).

1.2.4 Les objectifs et principes officiels informent et orientent le fonctionnement quotidien de l'établissement (Ontario 1).

1.2.5 Les objectifs et principes officiels sont clairement compris et mis en application par le personnel (Ontario 1). 
1.2.6 Quels que soient les divers objectifs poursuivis, on répond toujours aux besoins normaux du jeune, i.e. aux besoins communs à tout adolescent (cf. Québec 1, Etats-Unis 7, États-Unis 8, États-Unis 9).

1.2.7 L'établissement inclut, parmi ses objectifs, la valorisation du jeune, l'augmentation de son estime de soi en lui faisant connaître des succès (cf. Etats-Unis 8, Etats-Unis 9).

\subsection{Principes}

1.3.1 L'établissement accepte et met en application le principe de l'intervention minimale.

Définition : L'intervention minimale représente une règle selon laquelle on intervient le moins possible dans la vie d'un jeune, on choisit la mesure la moins contraignante possible et seulement s'il est évident qu'une intervention s'impose (cf. Québec 1, Wisconsin 1, et États-Unis 9).

1.3.2 L'établissement accepte et met en application le principe de la normalisation.

Définition : La normalisation peut être définie comme l'utilisation des moyens les plus normaux (couramment utilisés ou à tout le moins acceptés par la communauté), pour susciter des comportements les plus normaux possibles compte tenu du milieu culturel.

Le principe de la normalisation a aussi des implications dans les secteurs suivants :

- rythme des journées et des années

- espace et sites

- cycles de croissance, de vie

- possibilité de choisir, d'avoir des préférences au fil de la vie quotidienne (Ontario 1, et États-Unis 9).

1.3.3 L'établissement assure la continuité de ses services et ne cesse d'intervenir qu'après s'être assuré que le jeune reçoit ce dont il a besoin dans sa famille, dans un autre établissement ou ailleurs.

\subsection{Droits}

1.4.1 L'établissement possède un document sur les droits de ses clients par lequel il reconnaît, entre autres, leurs droits à la vie privée, au libre exercice religieux, à être informés des décisions qui les concernent et à tout autre droit reconnu au citoyen ordinaire (cf États-Unis 9).

1.4.2 L'exercice de ces droits n'est pas limité ou empêché comme mesure punitive (cf. Etats-Unis 9).

\section{DÉCISIONS À L'ADMISSION}

\subsection{Principes d'admission}

2.1.1 Les décisions des responsables de l'admission se conforment au principe voulant qu'aucun jeune ne reçoive plus de surveillance et d'aide qu'il n'en a vraiment besoin (États-Unis 5).

2.1.2 Les décisions des responsables de l'admission se conforment au principe voulant qu'aucun jeune délinquant ne soit placé dans un milieu plus sécuritaire que le risque qu'il représente ne l'exige (États-Unis 5).

2.1.3 Un placement n'est jamais permis parce qu'il fait l'affaire de la famille, de l'école, de la police, de l'agence de service social ou de l'agent de probation et, à plus forte raison, un placement en centre securitaire (cf. Wisconsin 1). 
2.1.4 Un placement n'est jamais fait ou prolongé pour faire un diagnostic, une évaluation ou pour compléter un dossier (cf. Ontario 2, New Jersey 1).

2.1.5 Un placement ne se fait que s'il se révèle être la solution optimale au problème posé par le jeune (États-Unis 8).

2.1.6 La détention et le centre sécuritaire sont des mesures de dernier recours (États-Unis 3).

2.1.7 Tout placement est considéré comme temporaire et, quand c'est possible, la durée de séjour est fixée dès l'admission (États-Unis 8).

\subsection{Politiques d'admission}

2.2.1 L'établissement possède une politique écrite d'admission qui comprend :

- les critères d'admission

- les pouvoirs et responsabilités de ceux qui prennent les décisions

- les procédures d'admission

(cf. Etats-Unis 4).

2.2.2 La politique d'admission de l'établissement est élaborée en collaboration avec les agences de référence (cour, centres de services sociaux, etc.) (Wisconsin 2).

2.2.3 La politique d'admission est révisée au moins à tous les deux ans (Wisconsin 2).

\subsection{Assistance pendant le placement}

2.3.1 Pendant l'admission, les jeunes reçoivent de l'aide pour traverser la crise du placement et les problèmes que pose une telle rupture (Wisconsin 2, États-Unis 8).

2.3.2 Dans les centres de transition, l'aide pour traverser les moments de crise est disponible 24 heures par jour (cf. Etats-Unis 7 ).

\subsection{Détention}

\subsubsection{Critères d'admission :}

2.4.1.1 Ne sont admis en détention que les jeunes qui ont commis ou sont présumés avoir commis un acte délinquant et qui représentent un danger pour la communauté (cf. Wisconsin 1).

2.4.1.2 Ne sont jamais admis en détention :

- Les jeunes qui peuvent être contrôlés par leurs parents et/ou par un agent de probation

- Les jeunes qui, presque certainement, ne se sauveront ni ne commettront un nouveau délit en attendant la décision du tribunal

- Les jeunes délinquants et non-délinquants qui doivent être retirés de leur famille parce qu'ils $y$ sont en danger

- Les jeunes que la police veut interroger

- Les jeunes que les cliniciens et agents de probation veulent évaluer

- Les jeunes dont les parents sollicitent une mesure de détention Commentaire : La détention n'a jamais été conçue pour être une garderie (Wisconsin 1).

2.4.2 Pouvoirs d'admettre en détention :

2.4.2.1 Un jeune ne peut être admis en détention sans une autorisation spécifique d'un juge ou d'un responsable de l'admission du centre de détention (Wisconsin 2). 
2.4.2.2 Le personnel de la probation, des services sociaux ou tout autre personnel clinique n'est pas autorisé à ordonner une mesure de détention sur une base administrative (Wisconsin 1).

\subsubsection{Procédures :}

2.4.3.1 Les responsables de l'admission dans les centres de détention ont l'autorité et la responsabilité de :

- renvoyer la plainte quand elle ne concerne pas la délinquance ou qu'elle est mineure, ou que les circonstances sont telles qu'aucune intervention n'est nécessaire ;

- renvoyer les plaintes qui semblent arbitraires ou vindicatives ;

- réorienter le plus grand nombre possible de jeunes vers une solution alternative (cf. Etats-Unis 5).

2.4.3.2 Des responsables d'admission dans les centres de détention sont disponibles 24 heures par jour, 7 jours par semaine, pour filtrer les admissions en détention (Wisconsin 2).

2.4.3.3 Les responsables de l'admission dans les centres de détention ont des politiques écrites et des critères pour référer les jeunes vers des solutions alternatives (Wisconsin 1).

\subsection{Hébergement}

2.5.1 Critères d'admission :

2.5.1.1 Un jeune peut être admis en hébergement temporaire dans les conditions suivantes :

- il n'existe pas d'adulte fiable qui accepte la responsabilité du jeune et la libération de celui-ci, avec une sommation, n'est pas indiquée

ou

— l'hébergement est nécessaire pour protéger la santé ou la sécurité du jeune

ou

- l'hébergement est nécessaire pour assurer sa présence a la prochaine comparution

ou

- la condition physique ou mentale du jeune rend contre-indiquée une libération immédiate

ou

- l'enfant ne veut pas retourner à la maison et il n'existe pas d'adulte capable de le prendre en charge (cf. New Jersey 1, États-Unis 1).

2.5.1.2 $\mathrm{Ne}$ sont pas admis en hébergement temporaire les jeunes qui :

- sont sérieusement perturbés émotivement

- semblent sérieusement malades

- sont en détention pour avoir commis des crimes violents graves (New Jersey 1).

2.5.1.3 Un enfant ne peut être placé en hébergement temporaire s'il n'a pas 10 ans ou plus (Wisconsin 2).

2.5.1.4 L'hébergement temporaire n'est pas utilisé quand une famille d'accueil de transition est disponible et constitue une solution adéquate (New Jersey 1). 


\subsubsection{Procédures :}

2.5.2.1 Les jeunes placés en hébergement temporaire ont une comparution dans les 24 heures, excluant les congés et fins de semaine, afin de décider s'il s'agit d'un placement approprié (Wisconsin 1).

2.5.2.2 L'hébergement non sécuritaire ne dépasse pas 30 jours (Wisconsin 1).

Commentaire : L'hébergement est une mesure de transition temporaire. L'expérience démontre que, quand une limite de temps est fixée à un placement, les services et les décisions viennent plus rapidement (cf. Wisconsin 1).

\section{DÉCISIONS EN COURS DINTERVENTION}

\subsection{Plan individuel d'intervention}

\section{Définition :}

Le plan individuel d'intervention est un plan d'action écrit, destiné à adapter les interventions aux besoins du client. Il est élaboré et modifié à intervalles réguliers avec la participation des personnes concernées. Il fixe des objectifs, identifie un continuum de développement, esquisse les étapes projetées et entrevoit les conséquences des interventions sur le développement du sujet (cf. Etats-Unis 9).

\subsubsection{Nature :}

3.1.1.1 L'établissement élabore pour chaque client un plan individuel d'intervention qui spécifie les objectifs à réaliser et les étapes à franchir (États-Unis 9).

3.1.1.2 Le plan initial d'intervention est formulé dans les 30 jours après l'admission du jeune (États-Unis 9).

3.1.1.3 Le plan individuel d'intervention repose sur des évaluations $d u$ jeune dans les secteurs suivants :
- social
- éducatif
— criminologique
- médical
- affectif
- cognitif (cf. Alabama 1 et Etats-Unis 9).

3.1.1.4 Le plan individuel d'intervention est écrit simplement et dans des termes compréhensibles pour tous ceux qui sont concernés (ÉtatsUnis 9).

\subsubsection{Objectifs du plan individuel d'intervention :}

3.1.2.1 Le plan individuel d'intervention contient des objectifs déterminés à partir de l'évaluation du jeune (cf. États-Unis 9).

3.1.2.2 Les objectifs du plan individuel d'intervention sont :

- énoncés séparément

- situés dans une séquence temporelle

- exprimés en termes comportementaux qui fournissent des indices mesurables de progrès (États-Unis 9).

\subsubsection{L'intervention :}

3.1.3.1 Le plan individuel d'intervention mise sur les forces et les qualites du jeune pour l'aider à se développer et à faire l'expérience du succès (cf. Québec 1). 
3.1.3.2 Le plan individuel d'intervention spécifie les modes d'intervention qui permettront de réaliser les objectifs énoncés (États-Unis 9).

3.1.3.3 Le plan individuel d'intervention décrit les conditions, activités ou obstacles susceptibles de nuire à la réalisation des objectifs (ÉtatsUnis 9).

3.1.3.4 Lintervention qui porte sur des problèmes spéciaux de l'enfant ne fait pas négliger pour autant les besoins normaux de tout jeune (Ontario 2).

3.1.3.5 Le plan individuel d'intervention identifie les agences extérieures capables de donner les services qui ne peuvent être fournis à l'interne (États-Unis 9).

3.1.3.6 Le plan individuel d'intervention identifie celui qui sera responsable de l'utilisation et de la coordination des services offerts par les différents praticiens de l'agence et par les autres établissements (Etats-Unis 9).

\subsubsection{Participation :}

3.1.4.1 Le plan individuel d'intervention est élaboré avec la participation :

- du jeune

- de ses parents

- du personnel de l'établissement concerné

- du personnel d'autres agences concernées par le client (ÉtatsUnis 9).

3.1.4.2 Le client et sa famille sont informés du contenu du plan individuel d'intervention (Québec 1, États-Unis 9).

3.1.4.3 Le plan individuel d'intervention comporte une entente écrite qui explicite le rôle et les responsabilités des personnes impliquées dans la réalisation du plan, y compris le jeune (cf. États-Unis 9).

\subsubsection{Révision :}

3.1.5.1 La révision du plan individuel d'intervention sert à :

- mesurer les progrès du jeune

- modifier les objectifs si nécessaire

-.. identifier les services dont le jeune a besoin

- identifier les mesures nécessaires pour contrecarrer ce qui bloque l'évolution du sujet

(États-Unis 9).

3.1.5.2 La révision du plan individuel d'intervention permet de décider de la fin du séjour, et du retour progressif du jeune dans la société (cf. Québec 1).

3.1.5.3 Le plan individuel d'intervention est révisé à tous les 3 mois (Etats-Unis 9).

\subsubsection{Conférence de cas :}

3.1.6.1 Lors de l'élaboration et de la révision du plan individuel d'intervention, on tient une conférence de cas à laquelle participent toutes les personnes concernées, y compris le jeune lui-même (cf. Québec 1).

3.1.6.2 Lors de la conférence de cas, une synthèse est faite des différents éléments du plan individuel d'intervention et des évaluations faites par des représentants de diverses spécialités (cf. Québec 1). 
3.2 Fin de séjour - transfert

3.2.1 L'établissement a une politique qui définit à quelles conditions le traitement prend fin (États-Unis 9).

3.2.2 Le transfert dans un autre établissement ou le retour dans la communauté n'est jamais retardé pour des raisons d'ordre administratif ou de recherche (cf. Québec 1).

3.2.3 Avant le départ du jeune d'un établissement, on organise une conférence de cas dans le but de :

- faire un bilan sur les effets de l'intervention

- préparer la sortie du jeune (cf. Québec 1).

3.2.4 Lors d'un transfert à un autre établissement, on assure la transition (Etats-Unis 9).

3.2.5 Lors d'hébergement temporaire, le personnel fait le maximum d'efforts pour retourner l'enfant dans sa famille et dans la communauté (New Jersey 1).

\subsection{Révision : Détention}

3.3.1 Un adolescent ne peut être détenu plus de 24 heures avant sa première comparution (États-Unis 1-3).

3.3.2 Si un jeune est maintenu en détention après une première comparution, on tient une comparution à toutes les deux semaines afin de décider de la nécessité de continuer la détention (cf. Québec 1 et Wisconsin 1).

\section{EDUCATION ET FORMATION SCOLAIRE}

\subsection{Principes}

4.1.1 Des services d'éducation sont disponibles pour tous les clients, quel que soit leur âge, leurs handicaps ou tout autre problème.

Définition :

Éducation : mesures pour favoriser le développement de l'individu aux niveaux intellectuel, sensori-moteur et affectif (États-Unis 8).

4.1.2 On offre aux jeunes la possibilité de continuer leur scolarité jusqu'à la fin du secondaire et/ou d'apprendre un métier (Wisconsin 1).

4.1.3 Le centre sécuritaire prévoit au moins trois heures d'activités académiques obligatoires par jour ou d'initiation au travail (orientation, apprentissage d'un métier, etc.) (Québec 1).

4.1.4 Les objectifs éducatifs embrassent à la fois le domaine académique et celui des interactions quotidiennes (États-Unis 9).

\subsection{Individualisation}

4.2.1 Les objectifs éducatifs définis spécifiquement pour chaque jeune rencontrent les normes suivantes :

- être basés sur une évaluation pertinente

- être formulés en termes comportementaux, facilitant ainsi leur application

- être réalisables

- se prêter à une évaluation continue

- changer selon les besoins du jeune (Etats-Unis 9).

\subsection{Contenu des programmes}

4.3.1 Les programmes d'éducation et de formation professionnelle rencontrent les normes officielles du ministère concerné (États-Unis 9). 
4.3.2 On met la disposition des clients des programmes éducatifs d'une qualité comparable à celle des étudiants du même âge dans les écoles publiques en ce qui a trait :

- au milieu physique

- aux qualifications du personnel

- à la durée quotidienne de l'enseignement

- à la durée de l'année scolaire

- à la taille de la classe

- au matériel scolaire

- aux services d'évaluation et autres services nécessaires (EtatsUnis 8).

4.4 Modalités

4.4.1 On envoie les jeunes à l'école publique lorsque les ressources locales le permettent et que les besoins du jeune sont satisfaits (États-Unis 8, New Jersey 1).

4.4.2 Des cours de rattrapage sont offerts aux jeunes qui en ont besoin (New Jersey 1).

\section{TRAVAIL - EMPLOI}

\subsection{Orientation}

5.1.1 L'établissement a un service ayant pour fonction de déterminer les aptitudes et le type de formation et de métier correspondant le mieux aux aptitudes du jeune (cf. Californie 9).

\subsection{Formation professionnelle}

5.2.1 L'ćtablissement aide le jeune dans le choix d'une formation professionnelle ou d'un travail spécifique (États-Unis 9).

5.2.2 Le programme de formation au travail a comme objectif de maximiser l'indépendance du client (États-Unis 9).

5.2.3 L'établissement développe un programme faisant partie intégrante du plan d'intervention pour faire acquérir au jeune la formation professionnelle choisie (cf. Californie 9).

5.2.4 Les objectifs du programme de formation et de travail sont établis en collaboration avec le jeune (États-Unis 9).

\subsection{Placement sur le marché du travail}

5.3.1 On établit au sein de l'agence ou en collaboration avec une autre agence un service de placement pour aider le jeune à se trouver un emploi (cf. Californie 9).

5.3.2 L'établissement intervient directement ou en utilisant des ressources extérieures, pour soutenir le jeune dans son adaptation au monde du travail (cf. États-Unis 9).

\section{LOISIRS}

\subsection{Politique}

6.1.1 L'établissement développe une politique des loisirs et considère ceuxci comme une partie intégrante de ses activités (cf. États-Unis 5).

\subsection{Objectifs}

6.2.1 Les loisirs prévus par l'établissement permettent de développer les talents et intérêts du jeune afin qu'il apprenne à faire un usage satisfaisant de ses temps libres (États-Unis 9). 
6.2.2 Les loisirs prévus par l'établissement procurent au jeune l'occasion de connaître le succès (États-Unis 9).

6.2.3 Les loisirs prévus par l'établissement offrent aux jeunes des expériences susceptibles de développer leurs aptitudes sociales (ÉtatsUnis 9).

6.2.4 Les loisirs prévus par l'établissement favorisent la santé physique et émotive (États-Unis 9).

6.3 Modalités

6.3.1 Le jeune est libre de participer ou non aux activités de loisir (cf. États-Unis 9).

6.3.2 On prévoit trois heures par jour pour les loisirs durant la semaine et 6 heures par jour durant la fin de semaine (Californie 3).

6.3.3 La gamme des activités offertes reflète la diversité des intérêts et des aptitudes des jeunes (États-Unis 5).

\section{TRAITEMENT}

\subsection{Politique}

7.1.1 L'établissement possède une politique sur l'usage des diverses formes de thérapie et, en particulier, sur les programmes de modification du comportement (cf. Etats-Unis 9).

7.1.2 Une telle politique spécifie, entre autres choses, les personnes ayant le pouvoir d'autoriser les thérapies, les mesures acceptables et prohibées (cf. États-Unis 9).

\subsection{Services de traitement offerts}

7.2.1 Il est possible pour le jeune d'avoir des psychothérapies individuelles avec une personne compétente lorsqu'il le désire (cf. États-Unis 1, Wisconsin 1).

7.2.2 Il est possible pour le jeune de participer à un programme de discussions ou de thérapies de groupe dirigé par des personnes compétentes (cf. États-Unis 5, Wisconsin 1).

7.2.3 Si nécessaire, on a recours à des techniques thérapeutiques spécialisées pour corriger les comportements inadaptés du jeune (cf. États-Unis 9).

7.2.4 Les services thérapeutiques spécialisés sont offerts dans le cadre de l'établissement ou à l'extêrieur de celui-ci (cf. États-Unis 9).

7.3 Libre choix

7.3.1 Les entrevues individuelles sont offertes aux jeunes sur une base volontaire (cf. Etats-Unis 5).

7.3.2 Les thérapies de groupe sont offertes aux jeunes sur une base volontaire (États-Unis 5).

7.3.3 Les jeunes qui refusent de participer aux entrevues individuelles ou aux thérapies de groupe ne sont pas pénalisés de ce fait (États-Unis 5).

\section{TRAVAIL AVEC LA FAMILLE}

8.1 Lien avec la famille

8.1.1 Dans la mesure du possible, on maintient des liens du jeune avec sa famille durant son séjour en institution (Wisconsin 1). 
8.2 Echanges d'informations avec la famille

8.2.1 On informe les parents du fonctionnement de l'agence, de ses règlements ainsi que des motifs justifiant le choix des activités et des interventions (cf. Californie 3).

8.2.2 On tient compte des connaissances et de l'opinion des parents lorsque des décisions importantes sont prises au sujet de leur enfant (Wisconsin 1).

\subsection{Participation de la famille}

8.3.1 On invite les parents à participer à l'élaboration du plan d'intervention de leur enfant (cf. Wisconsin 1).

\subsection{Formation du jeune dans sa famille}

8.4.1 L'établissement poursuit l'intervention auprès du jeune dans son milieu familial. Ceci s'inscrit dans le plan individuel d'intervention (Etats-Unis 9).

Commentaire : Il s'agit ici d'aider le jeune à s'adapter à son milieu familial par des interventions qui portent sur le jeune lorsqu'il se trouve dans sa famille. Ceci suppose que des professionnels de l'établissement se déplacent pour travailler dans la famille.

\subsection{Consultation sur l'éducation des enfants}

8.5.1 L'établissement offre à la famille un service de consultation sur l'éducation de leurs enfants (cf. États-Unis 9).

8.5.2 Le service de consultation sur l'éducation des enfants comprend les éléments suivants :

- consultation à domicile pour les parents

- observation, par le personnel spécialisé, de la conduite de l'enfant dans son milieu familial

- observation, par les parents, de l'enfant tel qu'il se comporte dans l'établissement

- discussion avec le personnel de ces observations (cf. Etats-Unis 9).

8.6 Services destinés à donner un répit d la famille

8.6.1 On donne aux parents la possibilité de jouir de périodes de répit dans la prise en charge de leur enfant par :

- un service de garderie

- un camp de vacances

- des activités de loisir pendant les soirées

- des activités de fin de semaine (cf. Etats-Unis 9).

8.7 Consultation sur les conflits familiaux

8.7.1 L'établissement offre un service de consultation pour aider le jeune et sa famille à résoudre les conflits interpersonnels ou les difficultés provenant de la désorganisation de la famille (cf. Etats-Unis 9).

8.8 Réinsertion dans la famille

8.8.1 Le travail auprès du jeune et de sa famille se poursuit lors du retour de celui-ci dans son milieu naturel (cf. États-Unis 9).

\section{REINSERTION SOCIALE}

\subsection{Objectifs}

9.1.1 L'intervention est orientée vers l'intégration du jeune dans le milieu social le plus propice à son développement (cf. États-Unis 9). 
9.1.2 Un service de réinsertion sociale aide le jeune à réintégrer avec succès sa famille (ọ un milieu de rechange), son école (ou un milieu de travail) et sa communauté (Ontario 2).

9.1.3 Les services de réinsertion sociale contribuent à raccourcir le séjour du jeune dans l'établissement (cf. Québec 1).

\subsection{Modalités}

9.2.1 Le service de réinsertion sociale :

- aide le jeune lors de son retour dans la société

- intervient auprès de la famille, des professeurs, des employeurs dans le but de faciliter l'intégration sociale du jeune

- réfère le jeune à d'autres services (cf. États-Unis 8).

9.2.2 Le service de réinsertion sociale aide les personnes qui côtoient l'enfant dans son milieu naturel (parents, professeurs, employeurs, etc.) à répondre aux besoins de celui-ci (Ontario 2).

\section{UTILISATION DES RESSOURCES EXTERNES}

\subsection{Politique}

10.1.1 L'établissement évite, dans la mesure du possible, de faire double emploi en créant des services qui existent déjà dans la communauté (cf. Etats-Unis 8, États-Unis 9, Wisconsin 1).

10.1.2 L'établissement utilise au maximum tous les services disponibles dans la communauté pour répondre aux besoins de ses clients (États-Unis 5).

10.1.3 On favorise les activités permettant les interactions sociales en dehors de l'établissement (Etats-Unis 9).

10.2 Types de services

10.2.1 Si l'établissement ne fournit pas à l'intérieur de ses cadres les services suivants, il voit à ce que le jeune les reçoive à l'extérieur :

— soins médicaux (incluant soins psychiatriques et dentaires)

- évaluation psychologique

- * counseling $*$ et/ou thérapie

- formation professionnelle

- placement sur le marché du travail

- rattrapage scolaire et/ou acquisition d'un diplôme

- tout autre service nécessaire au client ou au programme (cf. États-Unis 4).

10.2.2 L'établissement voit à fournir des soins médicaux de façon à :

- maintenir un niveau optimal de santé pour chaque pensionnaire

- prévenir les maladies (cf. États-Unis 8).

10.2.3 L'établissement a des ententes avec un ou plusieurs hôpitaux afin que des soins de première qualité soient assurés à ses pensionnaires 24 heures par jour (Québec 1, Wisconsin 1).

10.2.4 L'établissement ou l'agence surveille étroitement la qualité des soins psychiatriques fournis à ses clients (cf. Californie 7).

10.2.5 L'établissement facilite les rencontres entre le jeune et son avocat (cf. Wisconsin 1).

10.3 Informations sur les services externes

10.3.1 Il existe un service d'information pour les professionnels ou les agences ayant besoin d'aide. Ce service : 
- maintient un annuaire des ressources locales régionales

- établit des procédures pour obtenir, cataloguer et mettre à jour l'information (États-Unis 9).

\section{RELATIONS PERSONNEL-CLIENT}

\subsection{Rôle du responsable}

11.1.1 Chaque jeune est pris en charge par un responsable.

Définition : Le \& responsable » est un membre de l'agence qui est spécifiquement responsable d'un jeune donné. C'est le «parrain \$ de ce jeune, son «tuteur». Il a pour mission de coordonner l'ensemble des services dont le jeune peut avoir besoin. Il répond de la mise en ceuvre du programme individuel d'intervention et il défend les intérêts du jeune (cf. États-Unis 9).

11.1.2 La première fonction du responsable est de voir à ce que le programme individuel d'intervention soit respecté (États-Unis 9).

11.1.3 Le responsable voit à ce qu'on réponde aux besoins du jeune, en particulier dans les secteurs suivants :

- l'hébergement

- l'éducation

- l'emploi

- la santé

- les loisirs

- les relations avec la famille

- tout besoin spécial découlant des problèmes particuliers du jeune (cf. Etats-Unis 9).

11.1.4 Le responsable suit les progrès du jeune, il recueille les informations nécessaires et les fait parvenir à qui de đroit (cf. États-Unis 9).

11.1.5 Le responsable voit à ce que le dossier du jeune soit bien tenu et bien utilisé (cf. États-Unis 9).

11.1.6 Le responsable coordonne les divers services que reçoit le jeune (cf. Etats-Unis 9).

11.1.7 Le responsable a recours à des services internes ou externes à l'agence pour répondre aux besoins du jeune (cf. États-Unis 9).

11.1.8 Le responsable supervise le rendement et la qualité des divers services donnés au jeune (États-Unis 9).

11.1.9 Le responsable est le porte-parole du jeune et il défend les intérêts de celui-ci auprès de ceux qui lui dispensent des services spéciaux (cf. Etats-Unis 9).

11.1.10 Le responsable supporte le jeune tout au long de ses contacts avec l'agence (cf. États-Unis 9).

11.1.11 Le responsable facilite le transfert $d \mathfrak{u}$ jeune dans une autre agence ou un autre service (États-Unis 9).

11.1.12 Le jeune, sa famille et les membres du personnel connaissent le responsable du jeune (États-Unis 9).

\subsection{Choix du responsable}

11.2.1 Le jeune est transféré à un autre responsable ou service lorsque l'on réalise que le responsable éprouve de l'antipathie à l'égard du jeune (Québec 1).

11.2.2 Le client ou sa famille participe au choix du responsable (EtatsUnis 9). 
11.3 Qualité de la relation

11.3.1 Le personnel crée et maintient une atmosphère chaleureuse, propice au développement de sentiments positifs (États-Unis 9).

11.3.2 L'établissement fait tout en son pouvoir pour que les liens entre clients et personnel soient les meilleurs possible. On tient compte plus particulièrement des éléments suivants :

- communication honnête, ouverte, entre le personnel et les clients

- climat de respect mutuel entre personnel et jeunes

- sensibilité de la part du personnel aux besoins et émotions des jeunes

- reconnaissance et approbation des comportements montrant un développement social positif

- absence de la part du personnel de marques de mépris comme de ridicule (Californie 5).

\section{RĖGLEMENTS, SANCTIONS ET MESURES DE CONTRÔLE}

\subsection{Politiques}

12.1.1 L'établissement possède une politique précise sur l'usage des punitions, récompenses et mesures de contrôle (Québec 1).

12.1.2 L'usage des récompenses et punitions est étroitement contrôlé et supervisé par la direction de l'établissement (Québec 1).

\subsection{Règlements}

12.2.1 Les règlements peuvent être considérés comme les mesures les moins contraignantes pour réaliser les objectifs du centre ou pour préserver des valeurs essentielles (États-Unis 5).

12.2.2 Le choix des règlements est justifié par les raisons suivantes :

- répondre aux besoins du jeune

- protéger le jeune lui-même

- protéger les autres jeunes

- protéger le personnel

- protéger les citoyens en général (cf. États-Unis 5, Wisconsin 1).

12.2.3 Les règlements sont peu nombreux et portent sur l'essentiel (cf. Québec 1).

12.2.4 Les règlements sont accompagnés de l'éventail des sanctions susceptibles d'être infligées pour les violations (États-Unis 5, Wisconsin 1).

12.2.5 Les règlements sont suffisamment précis et spécifiques pour donner aux jeunes une connaissance suffisante sur ce qu'on attend d'eux (États-Unis 5, et Wisconsin 1).

12.2.6 Dès leur arrivée, les jeunes reçoivent une copie des règlements ainsi que les explications verbales nécessaires (New Jersey 1, Québec 1, Wisconsin 1).

12.2.7 Les jeunes participent à l'élaboration et à la révision des règlements (Québec 1).

12.2.8 Les règlements sont appliqués avec justice, uniformite, constance et sans arbitraire (cf. Québec 1).

\subsection{Sanctions}

12.3.1 Les sanctions prévues pour les violations au règlement sont proportionnelles à l'importance de la règle et à la gravité de la víolation (États-Unis 5). 
12.3.2 Les punitions pour violations mineures ne devraient pas dépasser la perte temporaire de privilèges dans le secteur des loisirs (Wisconsin 1).

12.3.3 Des politiques écrites interdisent :

- les châtiments corporels

- les mesures verbales qui risquent d'affecter l'estime de soi du sujet (insultes, étiquetage, surnoms, cris, humiliations, etc.)

- les mauvais traitements

- les punitions excessives

- les punitions de groupe

- les privations de repas

- la perte du courrier et des visites comme punitions

(cf. Californie 3, New Jersey 1, États-Unis 8, États-Unis 9, Wiscon$\sin 1)$.

Commentaire : Le châtiment corporel ne doit pas être confondu avec le droit du personnel de se protéger contre une attaque, ni avec l'exercice de la contrainte physique nécessaire pour empêcher le jeune de se blesser, ou de blesser les autres (Californie 3).

\subsection{Mesures de contrôle}

12.4.1 L'isolement n'est pas employé, sauf dans les circonstances suivantes :

- quand un jeune est incontrôlable et qu'il doit être retiré pour sa propre protection ou pour celle des autres et

- quand cette mesure fait partie d'un programme systématique de «time-out », réalisé selon les règles de l'art (cf. Californie 3, Etats-Unis 9 et Wisconsin 1).

\section{Définition :}

Isolement : le fait de placer un jeune seul dans une chambre fermée à clef.

12.4.2 Les jeunes en isolement sont sous observation directe et rencontrés par un membre du personnel au moins trois fois par jour (cf. États-Unis 8).

12.4.3 L'isolement, ainsi que toute autre mesure punitive d'importance font l'objet de rapports circonstanciés ; chaque fois qu'ils sont utilisés (Québec 1).

Commentaire : L'isolement est une mesure d'extrême urgence qui devrait être utilisée avec toute la parcimonie qui s'impose. À cette fin, les remarques suivantes peuvent être vues comme des guides de l'action :

- l'isolement ne devrait jamais durer plus longtemps que la période de crise l'ayant nécessité

- les conditions d'isolement ne devraient jamais être plus sévères que ne l'exige la situation ; à la limite, l'isolement peut n'être qu'un séjour du jeune dans sa chambre pour quelques minutes

- sous aucune condition, le jeune ne peut être privé de nourriture, de lumière, de la possibilité de dormir ou de se laver.

12.4.4 La médication n'est pas utilisée :

- comme punition

- par commodité

- comme un substitut à un programme

- en doses qui nuisent à la participation du jeune au programme (États-Unis 9). 


\subsection{Procédures}

12.5.1 Le personnel n'impose une punition qu'après avoir informé le client de la nature de son infraction et lui avoir donné l'occasion de s'expliquer ou de nier (Wisconsin 1).

12.5.2 Les jeunes n'appliquent pas de mesures disciplinaires à d'autres jeunes, sauf dans le cadre d'une démocratie de participation organisée en conformité avec des politiques écrites (États-Unis 9).

\section{MILIEU DE VIE}

\subsection{Vie de groupe}

13.1.1 Le personnel profite de la vie de groupe pour favoriser le développement du jeune à la communication (cf. États-Unis 9).

13.1.2 L'organisation de la vie de groupe favorise l'autonomie, l'indépendance, la solidarité et la participation démocratique (cf. New Jersey 1).

13.1.3 L'établissement procède périodiquement à une réévaluation de la qualité de la vie de groupe, compte tenu de ses objectifs (Québec 1).

\subsection{Vie privée}

13.2.1 Chaque pensionnaire peut se retirer dans un endroit calme, et privé quand il n'est pas spécifiquement engagé dans des activités structurées (États-Unis 8).

Commentaire : Il est essentiel que l'institution prévoit des périodes de la journée ainsi que des locaux pour que chaque adolescent puisse se retirer du groupe et jouir d'un peu de solitude. Les locaux en question peuvent être des salons, des chambres ou chambrettes.

13.2.2 Le centre de détention dispose de chambres individuelles (cf. Wisconsin 1).

\subsection{Mixité}

13.3.1 L'établissement organise des activités qui favorisent le développement de relations avec le sexe opposé (cf. Etats-Unis 8).

\subsection{Tâches quotidiennes}

13.4.1 Les jeunes doivent s'acquitter de certaines tâches quotidiennement (ménage, vaisselle, entretien, etc.).

Commentaire : il ne s'agit pas ici de faire porter tout le fardeau de l'entretien de l'établissement aux jeunes qui y résident, mais plutôt, en concordance avec le principe de normalisation, de faire en sorte que le pensionnaire assume des tâches similaires à celles dont il se chargerait dans le milieu familial (cf. New Jersey 1).

\subsection{Argent de poche}

13.5.1 Les jeunes disposent d'argent de poche (cf. New Jersey 1).

\subsection{Milieu physique}

13.6.1 Le milieu physique où vivent les jeunes favorise une atmosphère chaleureuse et détendue (Ontario 2).

\subsection{Accessibilité}

13.7.1 L'établissement est situé de telle sorte qu'il est facile d'avoir accès aux services de main-d'œuvre, médicaux, de loisir, etc. (cf. ÉtatsUnis 4). 


\section{CONTACTS AVEC L'EXTÉRIEUR}

\subsection{Principes}

14.1.1 Les jeunes ont le droit de communiquer et de correspondre avec les gens de leur choix aussi souvent qu'ils le veulent. Ce droit n'est restreint que s'il y a contre-indication évidente (cf. Wisconsin 1).

\subsection{Sorties}

14.2.1 L'établissement favorise les sorties comme moyen de préserver les liens entre le jeune et ses parents et de maintenir son enracinement social (cf. Wisconsin 1).

\subsection{Visites}

14.3.1 On prévoit, dans l'horaire, une période de visite pour les parents et autres personnes significatives au moins une fois par semaine (Californie 3).

14.3.2 L'horaire de visites des parents et autres personnes significatives est flexible et commode pour les visiteurs (cf. New Jersey 1).

14.3.3 Les parents et autres personnes significatives pour le jeune sont encouragés à visiter les unités de vie tout en respectant l'intimité des pensionnaires (États-Unis 8).

14.3.4 De l'espace dans l'unité de vie permet les conversations intimes entre le jeune et les visiteurs (non pas des pièces spéciales pour les visites) (États-Unis 8).

\subsection{Courrier}

14.4.1 Les autorités ne limitent pas le volume du courrier reçu et envoyé par le jeune (États-Unis 5, Wisconsin 1).

14.4.2 Le personnel ne lit ni ne censure le courrier reçu et envoyé. Il peut cependant inspecter le courrier s'il y a possibilité de contrebande (Wisconsin 1).

14.4.3 Seuls les produits défendus par la loi (ex : drogue) ou ceux qui menacent la sécurité de l'institution peuvent être considérés comme de la contrebande (Etats-Unis 5).

\subsection{Téléphones}

14.5.1 Les pensionnaires peuvent téléphoner à leurs parents et aux personnes significatives, sous réserve de considérations pratiques et de sécurité (cf. Wisconsin 1).

\subsection{Participation de la communauté à la vie de l'établissement}

14.6.1 Les autorités font venir des gens de l'extérieur pour maintenir les liens avec la communauté : parents, citoyens, volontaires, consultants, animateurs, groupes de loisir, etc., sont invités à participer aux activités de l'établissement (cf. Californie 6, États-Unis 5).

\section{PERSONNEL}

\subsection{Description des tâches}

15.1.1 L'établissement dispose d'une description écrite des tâches pour ses divers postes (aussi bien le personnel cadre que le personnel de base) (cf. États-Unis 8 et États-Unis 9).

15.1.2 Les descriptions des tâches sont précises, couvrent tous les champs d'activités et sont suffisamment détaillées pour permettre au person- 
nel d'avoir une idée claire de ses tâches et responsabilités (cf. Québec 1, États-Unis 7).

15.1.3 Les descriptions de tâches comprennent :

- les obligations

- les décisions à prendre

- les tâches à exécuter

- de qui relève l'employé

- le rôle de l'employé dans l'implantation du plan individuel d'intervention (cf. Californie 3).

\subsection{Embauche}

15.2.1 Le recrutement du personnel se fait à partir de la description des tâches des postes à combler (cf. États-Unis 8, Wisconsin 1).

15.2.2 À l'embauche, les candidats donnent en détails leurs antécédents et leurs qualifications pour travailler dans le poste sollicité. Ils apportent des informations complètes sur leur formation, leurs expériences et leurs emplois antérieurs, et sur leurs condamnations s'il y a lieu (cf. New Jersey 1).

15.2.3 L'embauche se fait à partir de la compétence du candidat sans discrimination quant au sexe, à la race, à la couleur, à la religion, à l'état civil ou à 'origine ethnique (cf. États-Unis 8, États-Unis 9).

15.2.4 L'établissement possède un noyau de professionnels spécialement formés ; cependant, il profite aussi des services d'employés qui, bien que sans qualification académique, ont une compétence adéquate et sont d'origine semblable à celle de la clientèle (cf. Wisconsin 1).

\subsection{Formation initiale}

15.3.1 Les employés nouvellement embauchés reçoivent au moins 80 heures d'initiation à leur travail et de formation sur place pendant leur première année de travail (Wisconsin 1).

15.3.2 La formation sur place comprend :

- une présentation des objectifs, principes et politiques de l'établissement

- une description des services offerts, de l'organisation et du fonctionnement de l'établissement

- une initiation aux méthodes d'intervention utilisées

- une étude des tâches du personnel concerné (cf. description de tâches)

- une initiation aux caractéristiques et aux besoins de la clientèle

- de l'information sur les agences avec lesquelles l'établissement coopère (cf. États-Unis 4, Êtats-Unis 8).

\subsection{Formation permanente}

15.4.1 L'établissement assure la formation permanente de son personnel (cf. États-Unis 7).

\subsection{Participation}

15.5.1 Le personnel participe effectivement aux décisions concernant l'établissement (cf. États-Unis 8).

15.5.2 Tous ceux qui travaillent dans l'établissement coopèrent et coordonnent leurs efforts pour réaliser les objectifs visés (cf. États-Unis 9). 


\subsection{Supervision}

15.6.1 Le personnel de base profite d'une période de supervision individuelle hebdomadaire au moins pendant la première année de travail (cf. Québec 1).

\subsection{Evaluation}

15.7.1 Le personnel affecté à la clientèle est évalué deux fois par année (cf. Québec 1, Etats-Unis 8, Etats-Unis 9).

15.7.2 L'évaluation de l'employé est :

- revue avec celui-ci

- incorporée à son dossier (Etats-Unis 8, Etats-Unis 9).

15.7.3 L'évaluation du personnel se fait avec l'aide d'un instrument qui :

- comporte une description détaillée des tâches

- est acceptée par l'employé et la direction

- permet de mesurer la qualité du travail réalisé par l'employé (cf. Etats-Unis 9).

15.7.4 Les nouveaux employés ont une période de probation d'au moins 9 mois pendant laquelle ils sont régulièrement supervisés et évalués (cf. Québec 1).

15.7.5 Les infractions présumées commises par des employés :

- font l'objet d'une enquête sérieuse

- les résultats de l'enquête sont consignés dans le dossier de l'employé

- des sanctions sont prises lorsqu'une infraction a effectivement été commise (cf. États-Unis 9).

15.8 Services essentiels

15.8.1 La direction et les syndicats se sont entendus sur les services essentiels à assurer aux jeunes en temps de crise (Québec 1).

15.8.2 Un personnel compétent est disponible pour s'occuper des enfants pendant la nuit et les fins de semaine (cf. New Jersey 1).

\subsection{Salaires}

15.9.1 Les membres du personnel reçoivent des salaires en rapport avec leur scolarité, formation et expérience (États-Unis 3).

15.9.2 Les salaires sont comparables aux salaires payés pour des tâches semblables dans le secteur scolaire ou la fonction publique (cf. Québec 1, États-Unis 3).

\subsection{Volontaires}

15.10.1 L'établissement utilise des volontaires pour compléter et soutenir les activités de son personnel permanent (États-Unis 9).

Commentaire : Les services rendus par les volontaires peuvent comprendre : l'organisation d'activités de loisir, sportives, religieuses, l'enseignement, l'initiation aux métiers, l'information sur le marché du travail, le transport, les voyages, vacances, visites, l'évaluation psychologique, thérapies spéciales, etc. (cf. Etats-Unis 8).

15.10.2 Les volontaires reçoivent une formation sur la nature et le sens de leur travail ainsi que sur leurs responsabilités (cf. Wisconsin 1).

15.10.3 Le travail des volontaires s'inscrit dans les objectifs de l'établissement et répond aux besoins des jeunes (cf. Californie, Etats-Unis 8). 


\section{DOSSIERS - INFORMATIONS}

\subsection{Qualité - pertinence}

16.1.1 Le dossier du jeune est maintenu à jour de façon à :

- développer et réviser le plan individuel d'intervention

- favoriser l'échange d'informations entre les différentes personnes responsables du jeune

- enregistrer les progrès dans la poursuite des objectifs du plan individuel d'intervention

- servir de point d'appui pour l'évaluation et la révision des services offerts par l'agence ou l'établissement

- fournir des données pour la recherche et la formation (ÉtatsUnis 9).

16.1.2 L'évaluation du jeune inscrite au dossier comprend non seulement un compte rendu des problèmes qu'il pose mais de plus, ses capacités, ses intérêts et toutes les ressources personnelles sur lesquelles on peut tabler pour l'aider (Québec 1, Ontario 2).

16.1.3 On ne met dans le dossier que l'information nécessaire, pour apporter des services adéquats au jeune (cf. Wisconsin 1, Ontario 2).

16.1.4 Les responsables s'assurent que toute information inscrite au dossier est exacte, complète et sans biais pouvant susciter de mauvaises interprétations (Wisconsin 1).

16.1.5 On bannit quelque forme d'étiquetage que ce soit dans les dossiers des clients (Québec 1).

\subsection{Forme}

16.2.1 Le dossier d'évaluation des jeunes est standardisé et uniformisé, l'organisation de ce dossier est conçue de telle façon que les principales données puissent permettre l'usage de l'informatique (Québec 1, Ontario 2).

16.2.2 Les données et informations contenues dans le dossier sont écrites dans un langage précis, clair et pouvant être compris par toutes les personnes susceptibles de l'utiliser (cf. États-Unis 9).

16.2.3 Toutes les données et informations au dossier sont datées et initialées par la personne les y ayant versées (cf. Etats-Unis 9).

\subsection{Diffusion}

16.3.1 Le contenu du dossier est communiqué aux parents et au jeune dans un langage clair et compréhensible (cf. Ontario 2, Québec 1).

16.3.2 Les parents et l'adolescent ont accès au dossier sur simple requête (cf. États-Unis 9, Wisconsin 1).

16.3.4 Les parents ou l'adolescent ont le droit de demander des rectifications de ce qu'ils jugent être des erreurs dans le contenu du dossier (cf. Wisconsin 1).

16.3.5 Lors d'un transfert d'un établissement ou d'un service à un autre, on transmet toutes les informations disponibles au service dorénavant chargé du jeune (cf. Québec 1, Californie 2).

\subsection{Accès et confidentialité}

16.4.1 L'établissement d'une politique pour déterminer qui peut avoir accès au dossier (cf. États-Unis 9).

16.4.2 La personne demandant accès au dossier doit justifier une telle requête (États-Unis 9). 
Commentaire : II s'agit ici de personnes autres que celles responsables du jeune (parents, éducateurs) ou le jeune lui-même.

16.4.3 Les dossiers sont confidentiels et le personnel n'en révèle le contenu ou n'en permet l'usage sans une autorisation officielle (New Jersey 1).

16.4.4 Lorsque les dossiers sont utilisés à des fins de recherche ou d'enseignement, les personnes les utilisant s'engagent formellement à respecter la confidentialité de Ieur contenu (cf. États-Unis 9, Wisconsin 1).

\section{COORDINATION AVEC LE RESEAU}

\subsection{Politiques}

17.1.1 L'établissement collabore avec les autres agences du réseau et leur fournit l'information nécessaire à leur travail auprès des jeunes (cf. Wisconsin 1).

17.1.2 La définition des objectifs de l'établissement se fait de concert avec l'ensemble des organismes d'une région donnée (cf. Québec 1).

17.1.3 La coordination avec les autres agences est organisée sur une base stable (États-Unis 9).

17.1.4 L'établissement maintient une étroite collaboration avec les autres services communautaires afin d'assurer les meilleurs services possibles pour les jeunes (New Jersey 1).

\subsection{Services}

17.2.1 L'établissement établit des liens de coopération avec les organisations ou agences communautaires importantes, parmi lesquelles :
a) Agence de main-d'œuvre
b) Commissions scolaires
c) Service social
d) Appareil de justice
e) Autres services communautaires : récréatifs, religieux, etc. (États-Unis 5).

17.2.2 L'établissement renseigne les tribunaux (juges, officiers de probation, avocats...) sur les services qu'il offre (cf. Etats-Unis 5).

17.2.3 L'établissement renseigne les services sociaux sur les services qu'il offre (cf. États-Unis 5).

\section{RECHERCHE ET EVALUATION}

\section{1 Évaluation}

18.1.1 L'agence évalue périodiquement sa performance en regard de ses objectifs initiaux.

18.1.2 L'évaluation régulière de l'agence porte sur les points suivants :

- les objectifs de l'établissement

- la relation entre les objectifs du service et les objectifs spécifiés dans le plan individuel d'intervention

- la relation entre les objectifs et les moyens utilisés

- la méthodologie des plans individuels d'intervention

- la performance du personnel (cf. États-Unis 9, Ontario 2).

18.1.3 Les résultats de l'évaluation conduisent à une révision systématique par le personnel et les administrateurs afin de déterminer :

- l'efficacité des programmes à rencontrer les buts poursuivis par l'agence 
- la nécessité de modifier ou d'ajouter aux programmes déja existants

- la nécessité de modifier les objectifs ou politiques déjà existants (cf. Etats-Unis 9, États-Unis 4).

18.1.4 L'agence collabore avec d'autres organismes afin d'évaluer la qualité de ses méthodes ou programmes et d'approfondir sa compréhension des problèmes de ses clients (États-Unis 7).

18.2 Recherche

18.2.1 En aucun cas on ne force un jeune, par la menace ou autrement, à participer à un programme de recherche (cf. États-Unis 9).

18.2.2 En aucun cas on ne retarde le transfert d'un jeune, son retour à la communauté ou son droit à tout autre privilège pour des motifs de recherche (cf. Etats-Unis 9, Québec 1).

\section{BIBLIOGRAPHIE}

Alabama 1 :

Alabama Youth Services Board (1975), Minimum Standards for Residential Facilities and Programs, Department of Youth Services, Alabama, U.S.A. 32 p.

\section{Californie 1:}

California Youth Authority (1973), Case Review and Planning : A Study of Treatment Goal Emphases and Consistency, California, U.S.A., $31 \mathrm{p}$.

Californie 2 :

California Youth Authority (1973), Education, Training and Deployment of Staff : A Survey of Probation Departments and the California Youth Authority, California, U.S.A., 110 p.

Californie 3 :

California Youth Authority (1973), Standards for Juvenile Halls, California, U.S.A., 32 p.

Californie 4 :

FISHMAN, D. (1973), « Creating and Supporting a Treatment Environment », California Youth Authority Quarterly, vol. 25, no 4, p. 4-7.

\section{Californie 5 :}

LEWIS, W.P. (1973), « Counseling Programs in Juvenile Institutions 》, California Youth Authority Quarterly, vol. 25, no 4, p. 8-12.

\section{Californie 6:}

FAUCHIER, D. (1973), «Ward Advisory Committees 》, California Youth Authority Quarterly, vol. 25, no 4, p. 13-16.

\section{Californie 7 :}

GOLDEN, S. (1973), Treatment Programs for the Emotionally Disturbed Correctional Client », California Youth Authority Quarterly, vol. 25, no 4 , p. 17-25. 
Californie 8 :

O'HARE, P. (1973), < Establishing Standards for Education Programs 》, California Youth Authority Quarterly, vol. 25, no 4, p. 38-44.

\section{Californie 9 :}

KUHL, C. (1973), * Vocational Educational Programs *, California Youth Authority Quarterly, vol. $25, \mathrm{n}^{\circ} 4$, p. 45-48.

New Jersey 1 :

- (1975), A Manual of Standards for Shelters Accepting Juveniles Acting Court Disposition, Department of Institutions and Agencies, New Jersey, U.S.A.

Ontario 1:

Ontario Association of Children's Mental Health Centers (1976), Report of the Task Force on Standards, Ontario, Canada.

Ontario 2 :

Ontario Association of Children's Mental Health Centers (1976), A Draft of Standards, Ontario, Canada.

Québec I :

- (1975), Rapport du comité d'études sur la réadaptation des enfants et adolescents placés en centres d'accueil, Ministère des Affaires sociales, Québec, Canada, 174 p.

U.S.A. 1 :

National Advisory Commission on Criminal Justice Standards and Goals (1973), Courts, U.S. Government Printing Office, 358 p.

U.S.A. 2 :

American Correctional Association (1959), Manual of Correctional Standards, U.S. Government Printing Office.

U.S.A. 3 :

Law Enforcement Assistance Administration (1975), Compendium of Model Correctional Legislation and Standards, U.S. Department of Justice, U.S. Government Printing Office.

U.S.A. 4 :

Law Enforcement Administration (1973), Guidelines and Standards for Halfeway Houses and Community Treatment Centers, U.S. Department of Justice, U.S. Government Printing Office, 273 p.

U.S.A. 5 :

National Advisory Commission on Criminal Justice Standards and Goals (1973), Corrections, U.S. Government Printing Office, 636 p.

U.S.A. 6 :

Child Welfare League of America (1971), A National Program for Comprehensive Child Welfare Services, New York, U.S.A., 48 p.

U.S.A. 7 :

Child Welfare League of America (1973), Standards for Child Protective Services, New York, U.S.A. 85 p. 
U.S.A. 8 :

Joint Commission on Accreditation of Hospitals (1975), Standards for Residential Facilities for the Mentally Retarded, Chicago, U.S.A. 159 p.

U.S.A. 9 :

Joint Commission on Accreditation of Hospitals (1973), Standards for Community Agencies Serving Persons with Mental Retardation and Other Developmental Disabilities, Chicago, U.S.A.

U.S.A. 10 :

MILLER H.L. (1977), * The \& Right to Treatment \& Can the Courts Rehabilitate and Cure s, The Public Interest, no 46, p. 97-118.

Wisconsin 1:

Special Study Committee on Criminal Justice Standards and Goals (1975), Juvenile Justice Standards and Goals, Wisconsin Council on Criminal Justice, U.S.A. 211 p.

Wisconsin 2 :

- (1976), Juvenile Detention in Wisconsin, Department of Health and Social Services, Wisconsin, U.S.A., 170 p. 